FORUM 2018 $33: 124$

https://doi.org/10.1007/s12312-018-0399-y

Online publiziert: 27. Februar 2018

(c) Springer Medizin Verlag $\mathrm{GmbH}$, ein Teil von Springer Nature 2018

\section{Gunter Schuch ${ }^{1}$ Peter Bannas ${ }^{2}$}

' HOPA Zentrum für Ambulante Onkologie im Struensee-Haus, Hamburg, Deutschland

${ }^{2}$ Klinik u. Poliklinik für Diagnostische u. Interventionelle Radiologie u. Nuklearmedizin, Zentrum für

Radiologie und Endoskopie, Universitätsklinikum Hamburg-Eppendorf, Hamburg, Deutschland

\title{
Lama-Antikörper helfen bei der Erforschung neuer Krebs- Therapie
}

Chemotherapie, Strahlentherapie und Operation sind die klassischen Behandlungsmethoden bei Krebs. Immer häufiger werden diese durch innovative Maßnahmen im Sinne von Immuntherapien ergänzt. Die Forschungsgruppe um Prof. Dr. Koch-Nolte und PD Dr. Bannas am Universitätsklinikum Hamburg-Eppendorf(UKE) erforscht zurzeit, inwiefern Antikörper, die aus Lamas gewonnen werden, im Kampf gegen eine seltene Krebs-Art nützlich sein können.

Für dieses Forschungsprojekt kooperiert das UKE mit der HämatologischOnkologischen Praxis Altona (HOPA). Gemeinsam wollen die beiden medizinischen Institutionen herausfinden, wie die Therapie mit Antikörpern - sogenannten Nanobodies - von Lamas bei Patienten anschlägt, die am Multiplen Myelom erkrankt sind. Hierbei handelt es sich um eine hämatologische Erkrankung, die sich im Knochenmark manifestiert und in deren Verlauf die Nieren, die Knochen sowie die Blutbildung des Patienten geschädigt werden können. Jedes Jahr erkranken in Deutschland etwa 6000 Menschen daran. Eine wirkungsvolle Heilungsmethode für diese Krankheit ist bisher nicht in Sicht.

„Die Kooperation zwischen dem UKE und der HOPA ermöglicht es, diese Krebs-Form besser zu erforschen", so Dr. Timon Hansen, Krebsspezialist in der HOPA. „Da es für seltene Erkrankungen nicht so viele Forschungsprogramme gibt, bedeutet dies eine große Hoffnung für die betroffenen Patienten.“

Die Mediziner konnten mit Hilfe von Lama-Antikörpern erste Fortschritte er- zielen, die die Behandlungschancen bei Multiplen Myelomen auf lange Sicht verbessern könnten. Da Nanobodies von Lamas kleiner als andere Antikörper sind, gelangen sie einfacher durch das Gewebe und können gezielt an die Krebszellen andocken und diese vernichten. Die ersten positiven Testergebnisse wurden an im Labor gezüchteten Krebszelllinien erreicht. Nun werden auch Untersuchungen mit menschlichem Knochenmark durchgeführt, welches von Patienten der HOPA zur Verfügung gestellt wurde, die hier wegen eines Multiplen Myeloms behandelt werden.

Im Verlauf des Jahres 2018 werden erste Studienergebnisse erwartet, auf deren Grundlage die Entwicklung neuer Immuntherapien möglich wird.

\section{Über die HOPA}

1976 gründete der Onkologe Prof. Ulrich Kleeberg mit der HOPA Deutschlands erste hämato-onkologische Praxis. Gewohntes Umfeld statt Krankenhaus ein damals höchst innovativer Ansatz, der das Leben mit der Erkrankung für viele Betroffene und ihre Angehörigen besser erträglich macht und Stabilität im Kampf gegen den Krebs gibt. Die HOPA ist Mitbegründerin der PalliativPartner Hamburg, einem Zusammenschluss von Ärzten und Pflegefachkräften, die die Patienten mit ihren komplexen Symptomen über die onkologische Behandlung hinaus zu Hause versorgen.

\section{Korrespondenzadresse}

\section{Gunter Schuch}

HOPA Zentrum für Ambulante Onkologie im Struensee-Haus

Mörkenstraße 47, 22767 Hamburg,

Deutschland

info@hopa-hamburg.de

\section{Peter Bannas}

Klinik u. Poliklinik für Diagnostische $u$. Interventionelle Radiologie u. Nuklearmedizin, Zentrum für Radiologie und Endoskopie, Universitätsklinikum Hamburg-Eppendorf Gebäude Ost 26, Martinistr. 52, 20246 Hamburg, Deutschland

p.bannas@uke.de

Interessenkonflikt. G. Schuch und P. Bannas geben an, dass kein Interessenkonflikt besteht. 